\title{
Article
}

\section{The Unit Re-Balancing Problem}

\author{
Robin Dee ${ }^{1, *}$, Armin Fügenschuh ${ }^{1}\left(\mathbb{D}\right.$ and George Kaimakamis ${ }^{2}$ \\ 1 Institute of Mathematics, Brandenburgische Technische Universität Cottbus-Senftenberg, \\ Platz der Deutschen Einheit 1, D-03046 Cottbus, Germany; fuegenschuh@b-tu.de \\ 2 Faculty of Mathematics and Engineering Sciences, Hellenic Army Academy, Varis-Koropiou Av., \\ 16673 Athens, Greece; gmiamis@sse.gr \\ * Correspondence: deerobin@b-tu.de
}

check for updates

Citation: Dee, R.; Fügenschuh, A.; Kaimakamis, G. The Unit

Re-Balancing Problem. Mathematics 2021, 9, 3205. https://doi.org/

$10.3390 /$ math 9243205

Academic Editor: Takayuki Hibi

Received: 28 October 2021

Accepted: 8 December 2021

Published: 11 December 2021

Publisher's Note: MDPI stays neutral with regard to jurisdictional claims in published maps and institutional affiliations.

Copyright: (c) 2021 by the authors. Licensee MDPI, Basel, Switzerland. This article is an open access article distributed under the terms and conditions of the Creative Commons Attribution (CC BY) license (https:// creativecommons.org/licenses/by/ $4.0 /)$.

\begin{abstract}
We describe the problem of re-balancing a number of units distributed over a geographic area. Each unit consists of a number of components. A value between 0 and 1 describes the current rating of each component. By a piecewise linear function, this value is converted into a nominal status assessment. The lowest of the statuses determines the efficiency of a unit, and the highest status its cost. An unbalanced unit has a gap between these two. To re-balance the units, components can be transferred. The goal is to maximize the efficiency of all units. On a secondary level, the cost for the re-balancing should be minimal. We present a mixed-integer nonlinear programming formulation for this problem, which describes the potential movement of components as a multi-commodity flow. The piecewise linear functions needed to obtain the status values are reformulated using inequalities and binary variables. This results in a mixed-integer linear program, and numerical standard solvers are able to compute proven optimal solutions for instances with up to 100 units. We present numerical solutions for a set of open test instances and a bi-criteria objective function, and discuss the trade-off between cost and efficiency.
\end{abstract}

Keywords: re-balancing problem; efficiency; mixed-integer linear programming; bi-criteria optimization

\section{Introduction}

In recent years, many nations tried to re-organize the structure of their public security forces (army, police, fire departments, first aids, etc.) in order to have better readiness and to be more efficient (see, for example, in [1]). At the same time, this re-organization has to lead to new structures that have to be used with the same or even lower budget.

The basic idea of this re-organization is to re-balance the force, manpower, and equipment by moving some supporting capabilities from the reserve component to the active component. The aim is to bring the reserve component force structure into closer alignment with that of the active component. The purpose of this re-balancing is to reduce the size of the institutional forces - the sustaining base-to an unprecedented low percentage of the total forces.

How do things get un-balanced? Any security force with its units can be found on the hierarchical level of an "organization" in the Living Systems Theory (LST) according to Miller [2]. LST is complemented by the Social Entropy Theory (SET), see Bailey [3] and references therein. SET describes social systems using PILOTS, which represent six macrosociological variables: population size $(\mathrm{P})$, information (I), level of living $(\mathrm{L})$, organization $(\mathrm{O})$, technology $(\mathrm{T})$, and space or territory $(\mathrm{S})$. Together, they describe the current status of a system. Both LST and SET are receptive for the concept of entropy, which has been originally developed for physical phenomena (the second law of thermodynamics) by Boltzmann in 1866 and later carried over to information theory by Shannon in 1949. In the context of LST and SET, it means that social systems tend to increase their state of disorder when they are not purposely organized by some outside force, up to the point when all system variables go to zero, which would indicate system death and maximum 
entropy. In this case, the system would not be able to utilize technology, or to organize, or to occupy and defend space [3]. This status clearly needs to be avoided for security forces. The re-balancing that we describe in our work can be seen as an external force to get things back in order before entropy raises to an unhealthy level.

In this paper, we describe a mathematical model which yields a suggestion to the Greek military on how to re-balance its units. We have implemented the model with army sections in mind but it is easily adapted for other nations and forces (police, fire departments, first aids, etc.). The proposed model is capable to re-organize the units in order

(i) to have the operational capability to execute their core mission,

(ii) to have the ability to manage the flexibility,

(iii) to have the required strength and efficiency, and

(iv) to remain within planned budget.

Re-balancing type problems received a lot of attention from researchers from various scientific fields.

Many applications from an economic perspective have already occurred which used the re-balancing problem in order to construct hedged portfolios or find the optimal portfolio under certain restrictions $[4,5]$. The so-called portfolio re-balancing problem deals with adjusting the portfolio with the goal of either maximizing the expected return or minimizing the risk of the portfolio [6].

There are a few applications of re-balancing in logistics such as the bike re-balancing problem $[7,8]$, in which the main issue is to re-distribute shared bikes with the objective of minimizing the total cost. A similar problem exists for the re-location of vehicles at car-sharing companies, see in $[9,10]$.

Some authors used the notion of re-balancing in order to address problems in predictive regression, for example, in [11].

In computer science, re-balancing is used to solve problems concerning several aspects such as search trees, assignment problems, or load re-balancing problems [12,13]. In the load re-balancing problem, the load consists of a set of tasks, associated with a processing time, and should be balanced on a set of machines. In [14], the authors study the load re-balancing problem as a game.

Balancing resources is essential in many aspects of public service, e.g., balancing the workload of workers. In [15], the authors focus on locating and re-locating a fleet of response units (from army, police, etc.) in a specific transportation network to optimally cover a number of facilities. They present the problem as a mixed-integer linear program and develop a heuristic algorithm. In [16], the authors consider a multi-commodity re-balancing and transportation problem with traffic congestion in disaster response. This problem is formulated as a bi-objective stochastic mixed-integer nonlinear program and approached via linearization and epsilon-constraint methods. Another well known application of the re-location (re-balancing) problem is the one that deals with emergency vehicles $[17,18]$. For vehicle routing problems dealing with specialized technicians, balancing issues in the workload of technicians were modeled in [19] and solved with a bi-objective optimization model [20]. In a military context, planning flights and maintenance of military aircrafts is considered in [21], where flights must be balanced to keep aircrafts intact as long as possible and maintenance operations must be balanced to keep the squadron available at all times. The authors improved programming model by training a Machine Learning model on a large set of past real-world instances.

The use of the term re-balancing as we want to understand it has to do with the dynamics. We use this term instead of re-location because in the case where we have some army units we want to re-balance their critical different components (force, manpower, and equipment) from one to another unit in order to increase the efficiency. In this framework the term re-balancing is more appropriate than re-location as re-location deals mainly with finding the best location for each unit. Our approach, for army units, deals with all the components that an army unit has and characterizes them. With the help of piecewise linear functions, the effect of shifting components (possibly leaving single units under- or 
overequipped) can be represented more detailed and the status of different components can be compared. With our work presented in this paper, we are able to fit nonlinear correlations (e.g., the efficiency and cost of an army unit depending on various ratings) into a mixed-integer linear programming model via the usage of piecewise linear functions. This offers a new perspective on re-balancing problems.

The remainder of this paper is organized as follows. In Section 2, we present a first mathematical model with variables, an objective function, and restrictions, including a first example instance. This basic model is applicable to real-world problems. In Section 3, we introduce an advanced model as an alternative draft to Section 2. Section 4 contains computational results with which the performance of different numerical solvers is analyzed, including an instance inspired by real-world data which shows how the current-day situation can be improved with the help of our models. Finally, in Section 5, we provide some concluding remarks.

\section{A Basic Model}

A unit in any public security force can be characterized by its components. In our presentation we keep the focus on army units. They can be characterized by their amount of armor, personnel, and equipment. By rating each characteristic in every unit with a number between 0 and 1, we can assign statuses. For example, armor could need a high rating to reach a high status, whereas personnel could reach a satisfying status even with a low rating. To determine the cost and efficiency of a single unit, we compare its status in all characteristics. An example is shown in Figure 1. The straight lines represent the status of the unit in the respective characteristics-the longer the line, the higher the status. The cost to maintain a unit depends on its maximum status among its characteristics (depicted as the outer circle on the left). In an analogous manner, the efficiency value of a unit is dependent on its minimum status among its characteristics (inner circle on the left). It follows that it would be optimal to have three equal status values as shown on the right.

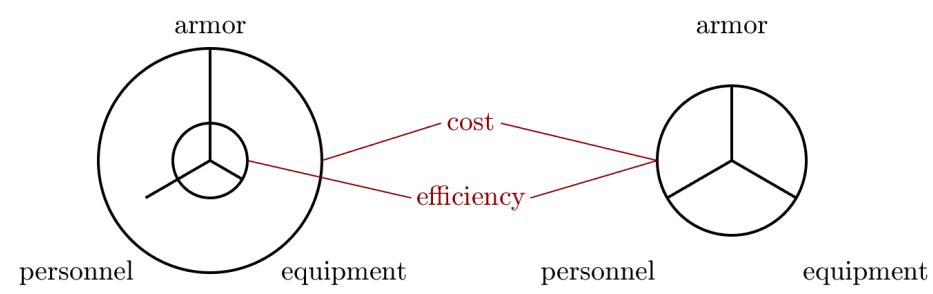

Figure 1. Determination of cost and efficiency of an un-balanced (left) and balanced unit (right).

\subsection{Sets}

An organization such as an army consists of several units. These units are numbered in the set $\mathcal{U}:=\{1, \ldots, U\}$, index $u$. Every unit can be described by various characteristics which are included in the set $\mathcal{C}:=\{1, \ldots, C\}$, index $c$. Depending on how well a unit can provide each of the characteristics, a status out of $\mathcal{S}:=\{1, \ldots, S\}$, index $s$, is assigned; 1 being the worst and $S$ being the best possible status. The total area on which the units are distributed, e.g., a country, is divided into regions, e.g., counties or islands. The set of regions is defined as $\mathcal{R}:=\{1, \ldots, R\}$, index $r$.

\subsection{Parameters}

The current ratings of all units (i.e., before the optimization) are gathered in a matrix $\left(p_{c, u}^{0}\right)_{c, u}$ with entries in $[0,1]$. Every entry of this matrix can be attached to a status: Each status belongs to an interval range $\left[\underline{b}_{c, s}, \bar{b}_{c, s}\right]$ with $0 \leq \underline{b}_{c, s} \leq \bar{b}_{c, s} \leq 1$. The parameter $\xi_{u, c, s}^{0} \in\{0,1\}$ describes if characteristic $c$ of unit $u$ is in status $s$ or not $\left(\xi_{u, c, s}^{0}=1\right.$ meaning that characteristic $c$ of unit $u$ is in status $s$ ). Additionally, $\alpha_{u, s}^{0} \in\{0,1\}$ captures if status $s$ is the lowest of unit $u$ or not; similarly, $\beta_{u, s}^{0} \in\{0,1\}$ marks if status $s$ is the highest of unit 
$u$ or not. To be considered open, a unit needs a minimum rating of $\epsilon_{\text {open }} \in[0,1]$ in every characteristic. For every unit $u$, the parameter $x_{u}^{0} \in\{0,1\}$ defines if $u$ is open or not. For a fixed $u \in \mathcal{U}$ and $c \in \mathcal{C}$, precisely one value of $\xi_{u, c, S}^{0}$ for some $s \in \mathcal{S}$ is equal to one, if and only if the unit $u$ is open. If the unit is closed, all $\xi_{u, c, s}^{0}$ are equal to zero. Therefore, the parameters fulfill the equation

$$
x_{u}^{0}=\sum_{s \in \mathcal{S}} \xi_{u, c, s}^{0}, \forall u \in \mathcal{U}, c \in \mathcal{C} .
$$

Similarly, for the lowest and the highest status, the corresponding equations are

$$
x_{u}^{0}=\sum_{s \in \mathcal{S}} \alpha_{u, s}^{0}=\sum_{s \in \mathcal{S}} \beta_{u, s}^{0}, \forall u \in \mathcal{U}
$$

We assume that the given parameters fulfill certain conditions which define a feasible instance and which are checked when input data is given.

A unit is closed if it is rated zero in all characteristics:

$$
p_{c, u}^{0} \leq x_{u}^{0}, \quad \forall u \in \mathcal{U}, c \in \mathcal{C} .
$$

To be open, a unit needs to have every characteristic rated above a minimum value:

$$
\epsilon_{\text {open }} x_{u}^{0} \leq p_{c, u}^{0}, \quad \forall u \in \mathcal{U}, c \in \mathcal{C} .
$$

For each unit $u$ and each characteristic $c$, the status is coupled to the rating:

$$
\sum_{s \in \mathcal{S}} \underline{b}_{c, s} \cdot \xi_{u, c, s}^{0} \leq p_{c, u}^{0} \leq \sum_{s \in \mathcal{S}} \bar{b}_{c, s} \cdot \xi_{u, c, s}^{0} \quad \forall u \in \mathcal{U}, c \in \mathcal{C} .
$$

If the lowest status of a unit $u$ is $s$, then at least one characteristic must be in this status as well and no characteristic can be of status lower than $s$ :

$$
\begin{gathered}
\alpha_{u, s}^{0} \leq \sum_{c \in \mathcal{C}} \xi_{u, c, s}^{0}, \forall u \in \mathcal{U}, s \in \mathcal{S}, \\
\alpha_{u, s}^{0}+\xi_{u, c, s_{1}}^{0} \leq 1, \quad \forall u \in \mathcal{U}, c \in \mathcal{C}, s, s_{1} \in \mathcal{S}, s_{1}<s .
\end{gathered}
$$

If the highest status of a unit $u$ is $s$, then at least one characteristic must be in this status as well and no characteristic can be of status higher than $s$ :

$$
\begin{gathered}
\beta_{u, s}^{0} \leq \sum_{c \in \mathcal{C}} \xi_{u, c, s}^{0}, \quad \forall u \in \mathcal{U}, s \in \mathcal{S}, \\
\beta_{u, s}^{0}+\xi_{u, c, s_{1}}^{0} \leq 1, \quad \forall u \in \mathcal{U}, c \in \mathcal{C}, s, s_{1} \in \mathcal{S}, s_{1}>s .
\end{gathered}
$$

Let $k_{s} \in \mathbb{R}^{+}$be the cost coefficient and $v_{s} \in \mathbb{R}^{+}$be the efficiency coefficient for $s \in S$. As book-keeping parameters we introduce

$$
K^{0}=\sum_{u \in \mathcal{U}, s \in \mathcal{S}} k_{s} \cdot \beta_{u, s}^{0} \in \mathbb{R}^{+}, \quad V^{0}=\sum_{u \in \mathcal{U}, s \in \mathcal{S}} v_{s} \cdot \alpha_{u, s}^{0} \in \mathbb{R}^{+},
$$

which reflect the cost and efficiency of all units before the re-balancing, respectively.

Every unit has geographical coordinates $\left(X_{u}, Y_{u}\right) \in \mathbb{R}^{2}$ and is located in a region $r_{u}$ with $r_{u} \in \mathcal{R}$. For some regions, in particular capitals and smaller islands, it is desired to specify lower and upper bounds on the number of units in this region that have at least a particular efficiency value $s$, specified as $\underline{n}_{r, s}, \bar{n}_{r, s} \in \mathbb{N}$ with $0 \leq \underline{n}_{r, s} \leq \bar{n}_{r, s} \leq U$. Shipping costs vary between regions. Let $\gamma_{r_{1}, r_{2}} \in \mathbb{R}^{+}$be the shipping cost factor between regions $r_{1}$ and $r_{2}$. Different characteristics also have different shipping cost factors $\kappa_{\mathcal{c}} \in \mathbb{R}^{+}$. Total 
shipping costs are bounded by $\sigma_{\max } \in \mathbb{R}^{+}$. The Euclidean distance between two units is given by

$$
d: \mathcal{U} \times \mathcal{U} \rightarrow \mathbb{R}^{+},\left(u_{1}, u_{2}\right) \mapsto \sqrt{\left(X_{u_{2}}-X_{u_{1}}\right)^{2}+\left(Y_{u_{2}}-Y_{u_{1}}\right)^{2}} .
$$

\subsection{Variables}

During the re-balancing, the amount of characteristic $c$ that is transferred from unit $u_{1}$ to unit $u_{2}$ is stored in $f_{c, u_{1}, u_{2}} \in[0,1]$.

After the re-balancing, $p_{c, u} \in[0,1]$ notates the value of characteristic $c$ in unit $u$. In $x_{u} \in\{0,1\}$, we can see if unit $u$ is still open or not. Analogous to the paragraph above, $\xi_{u, c, s} \in\{0,1\}$ tells if characteristic $c$ of unit $u$ is in status $s, \alpha_{u, s} \in\{0,1\}$ tells if status $s$ is the lowest of unit $u$ and $\beta_{u, s} \in\{0,1\}$ tells if status $s$ is the highest of unit $u$.

The book-keeping variables $K \in \mathbb{R}^{+}$and $V \in \mathbb{R}^{+}$represent the cost and efficiency of all units "after", respectively.

A survey of the sets, parameters, and variables can be found in Table 1 .

Table 1. Sets, parameters, and variables used in the model formulation.

\begin{tabular}{|c|c|c|}
\hline Name & Definition & Description \\
\hline $\mathcal{U}$ & $\{1, \ldots, U\}$ & units \\
\hline $\mathcal{C}$ & $\{1, \ldots, C\}$ & characteristics \\
\hline $\mathcal{S}$ & $\{1, \ldots, S\}$ & statuses \\
\hline $\mathcal{R}$ & $\{1, \ldots, R\}$ & regions \\
\hline Name & Range & Description \\
\hline$p_{c, u}^{0}$ & {$[0,1]$} & rating of characteristic $c$ in unit $u$ \\
\hline$\underline{b}_{c, S}$ & {$\left[0, \bar{b}_{c, s}\right]$} & lower bound for interval of status $s$ in characteristic $c$ \\
\hline$\overline{\bar{b}}_{c, s}$ & {$\left[\underline{b}_{c, S}, 1\right]$} & upper bound for interval of status $s$ in characteristic $c$ \\
\hline$\xi_{u, c, s}^{0}$ & $\{0,1\}$ & Is characteristic $c$ of unit $u$ in status $s$ "before" (yes/no)? \\
\hline$\alpha_{u, s}^{0}$ & $\{0,1\}$ & Is status $s$ the lowest of unit $u$ "before" (yes/no)? \\
\hline$\beta_{u, s}^{0}$ & $\{0,1\}$ & Is status $s$ the highest of unit $u$ "before" (yes/no)? \\
\hline$x_{u}^{0}$ & $\{0,1\}$ & Is unit $u$ open "before" (yes/no)? \\
\hline$\epsilon_{\text {open }}$ & {$[0,1]$} & minimum rating in every characteristic to consider a unit open \\
\hline$k_{s}$ & $\mathbb{R}^{+}$ & cost coefficient of status $s$ \\
\hline$v_{s}$ & $\mathbb{R}^{+}$ & efficiency coefficient of status $s$ \\
\hline$K^{0}$ & $\mathbb{R}^{+}$ & cost of all units "before" \\
\hline$V^{0}$ & $\mathbb{R}^{+}$ & efficiency of all units "before" \\
\hline$\left(X_{u}, Y_{u}\right)$ & $\mathbb{R}^{2}$ & geographical coordinates of unit $u$ \\
\hline$r_{u}$ & $\mathcal{R}$ & region of unit $u$ \\
\hline$\underline{n}_{r, s}$ & {$\left[0, \bar{n}_{r, s}\right]$} & minimum number of units in region $r$ with efficiency at least $s$ \\
\hline $\bar{n}_{r, s}$ & {$\left[\underline{n}_{r, s}, U\right]$} & maximum number of units in region $r$ with efficiency at least $s$ \\
\hline$\gamma_{r_{1}, r_{2}}$ & $\mathbb{R}^{+}$ & shipping cost factor between regions $r_{1}$ and $r_{2}$ \\
\hline$\kappa_{c}$ & $\mathbb{R}^{+}$ & shipping cost factor for characteristic $c$ \\
\hline K & $\mathbb{R}^{+}$ & cost of all units "after" \\
\hline$V$ & $\mathbb{R}^{+}$ & efficiency of all units "after" \\
\hline$f_{c, u_{1}, u_{2}}$ & {$[0,1]$} & amount of characteristic $c$ transferred from unit $u_{1}$ to $u_{2}$ \\
\hline$p_{c, u}$ & {$[0,1]$} & value of characteristic $c$ in unit $u$ after re-balancing \\
\hline$x_{u}$ & $\{0,1\}$ & Is unit $u$ open "after" (yes/no)? \\
\hline$\xi_{u, c, s}^{u}$ & $\{0,1\}$ & Is characteristic $c$ of unit $u$ in status $s$ "after" (yes/no)? \\
\hline$\alpha_{u, s}$ & $\{0,1\}$ & Is status $s$ the lowest of unit $u$ "after" (yes/no)? \\
\hline$\beta_{u, s}$ & $\{0,1\}$ & Is status $s$ the highest of unit $u$ "after" (yes/no)? \\
\hline
\end{tabular}




\subsection{Objective and Constraints}

The goal is to maximize the total efficiency after re-balancing the units:

$$
\max V \text {. }
$$

The cost and efficiency "after" is computed (by adding up the lowest status of every unit multiplied with the corresponding cost value):

$$
\begin{aligned}
& K=\sum_{u \in \mathcal{U}, s \in \mathcal{S}} k_{s} \cdot \beta_{u, s}, \\
& V=\sum_{u \in \mathcal{U}, s \in \mathcal{S}} v_{s} \cdot \alpha_{u, s} .
\end{aligned}
$$

The budget is limited. The re-balanced units ("after") must not be more expensive than "before":

$$
K \leq K^{0}
$$

After the re-balancing, the following conditions must hold.

Every unit $u$ is either closed or in some status:

$$
x_{u}=\sum_{s \in \mathcal{S}} \alpha_{u, s}=\sum_{s \in \mathcal{S}} \beta_{u, s}, \quad \forall u \in \mathcal{U}
$$

Additionally, it is ensured that each active unit $u$ is in a unique status:

$$
x_{u}=\sum_{s \in \mathcal{S}} \xi_{u, c, s}, \forall u \in \mathcal{U}, c \in \mathcal{C} .
$$

A closed unit has to be emptied completely:

$$
p_{c, u} \leq x_{u}, \quad \forall u \in \mathcal{U}, c \in \mathcal{C} .
$$

An open unit must have a minimum amount of any characteristic:

$$
\epsilon_{\text {open }} \cdot x_{u} \leq p_{c, u}, \forall u \in \mathcal{U}, c \in \mathcal{C} .
$$

The existing units can be distributed (cannibalized) to other units:

$$
p_{c, u}^{0}=\sum_{u_{1} \in \mathcal{U}} f_{c, u, u_{1}}, \quad p_{c, u}=\sum_{u_{0} \in \mathcal{U}} f_{c, u_{0}, u}, \quad \forall u \in \mathcal{U}, c \in \mathcal{C} .
$$

For each unit $u$ and each characteristic $c$, the status is coupled to the rating:

$$
\sum_{s \in \mathcal{S}} \underline{b}_{c, s} \cdot \xi_{u, c, s} \leq p_{c, u} \leq \sum_{s \in \mathcal{S}} \bar{b}_{c, s} \cdot \xi_{u, c, s} \quad \forall u \in \mathcal{U}, c \in \mathcal{C} .
$$

If the lowest status of a unit $u$ is $s$, then at least one characteristic must be in this status as well and no characteristic can be of status lower than $s$ :

$$
\begin{gathered}
\alpha_{u, s} \leq \sum_{c \in \mathcal{C}} \xi_{u, c, s}, \quad \forall u \in \mathcal{U}, s \in \mathcal{S}, \\
\alpha_{u, s}+\xi_{u, c, s_{1}} \leq 1, \quad \forall u \in \mathcal{U}, c \in \mathcal{C}, s, s_{1} \in \mathcal{S}, s_{1}<s .
\end{gathered}
$$

If the highest status of a unit $u$ is $s$, then at least one characteristic must be in this status as well and no characteristic can be of status higher than $s$ :

$$
\begin{gathered}
\beta_{u, s} \leq \sum_{c \in \mathcal{C}} \xi_{u, c, s}, \quad \forall u \in \mathcal{U}, s \in \mathcal{S} \\
\beta_{u, s}+\xi_{u, c, s_{1}} \leq 1, \quad \forall u \in \mathcal{U}, c \in \mathcal{C}, s, s_{1} \in \mathcal{S}, s_{1}>s .
\end{gathered}
$$


Each region $r$ has a lower (e.g., capitals or large regions) and upper (e.g., smaller regions or islands) bound on the number of units with efficiency at least $s$ :

$$
\underline{n}_{r, s} \leq \sum_{\substack{u \in U \\ r_{u}=r}} \sum_{s_{1} \in \mathcal{S}:} \alpha_{s_{1} \geq s} \leq \bar{n}_{r, s}, \quad \forall r \in \mathcal{R}, s \in \mathcal{S} .
$$

Shipping costs are not allowed to exceed $\sigma_{\max }$ :

$$
\sum_{\substack{c \in C, u, u_{1} \in U}} \kappa_{\mathcal{c}} \cdot \gamma_{r_{u}, r_{u_{1}}} \cdot d\left(u, u_{1}\right) \cdot f_{c, u, u 1} \leq \sigma_{\max }
$$

The model consists of (12)-(27) plus integer and non-negativity constraints.

\section{A Continuous Model}

The basic model presented in the previous section can be extended to portray realworld scenarios more realistically. A drawback is the fact that the conversion from ratings to statuses is based on step functions (as depicted in Figure 2). As a consequence, characteristics are downgraded to barely hold their current status and not to "waste" any capacities. To counter this, we decided to convert the ratings to statuses via piecewise linear functions, thus turning the statuses into continuous variables.

\subsection{Sets}

Sets $\mathcal{U}, \mathcal{C}$, and $\mathcal{R}$ are adopted from Section 2. Furthermore, let $\mathcal{B}$, index $b$ with $0 \leq b \leq S_{\max }\left(S_{\max }\right.$ being the highest possible status) be the set containing all values for the regional restrictions described in Section 2.4 .

\subsection{Parameters}

The following parameters are adopted from Section 2: rating "before" $p_{c, u}^{0}$, minimum rating $\epsilon_{\text {open, }}$ activity "before" $x_{u}^{0}$ with the following inequalities:

$$
\begin{gathered}
p_{c, u}^{0} \leq x_{u}^{0} \quad \forall u \in \mathcal{U}, c \in \mathcal{C}, \\
\epsilon_{\text {open }} \cdot x_{u}^{0} \leq p_{c, u}^{0}, \quad \forall u \in \mathcal{U}, c \in \mathcal{C},
\end{gathered}
$$

coordinates $X_{u}, Y_{u}$, region $r_{u}$, regional shipping cost factor $\gamma_{r_{1}, r_{2}}$, characteristical shipping cost factor $\kappa_{c}$, maximum shipping costs $\sigma_{\max }$, Euclidean distance $d\left(u_{1}, u_{2}\right)$. The regional efficiency restrictions are now given as $\underline{n}_{r}(b), \bar{n}_{r}(b) \in \mathbb{N}$ with $0 \leq \underline{n}_{r}(b) \leq \bar{n}_{r}(b) \leq U$.

To determine the status of each characteristic in a unit, we apply continuous piecewise linear functions to $p_{c, u}^{0}$. This way, statuses can take any value in $\left[0, S_{\max }\right]$. Breakpoints of each piecewise linear function are given in the matrix $\left(g_{c, i}\right)_{c, i}, i \in\left\{1, \ldots, S_{\max }\right\}$, which contains the values of $p_{c, u}^{0}$ that correspond to an integer status value. It follows that $g_{c, S_{\max }}=1$ for all $c \in \mathcal{C}$. We also assume that $p_{c, u}^{0}=0$ results in a corresponding status of 0 .

Cost and efficiency of a unit are determined similarly. The vectors $g^{k}$ and $g^{v}$ contain the cost and efficiency values of the integer statuses $1, \ldots, S_{\max }$ and serve as breakpoints of the respective continuous piecewise linear functions. The statuses $s_{c, u}^{0}, \operatorname{cost} k_{u}^{0}$, and efficiency $v_{u}^{0}$ of a unit prior to the re-balancing can be calculated beforehand. The total cost and efficiency values "before" are computed as follows:

$$
K^{0}:=\sum_{u \in \mathcal{U}} k_{u}^{0} \in \mathbb{R}^{+}, \quad V^{0}:=\sum_{u \in \mathcal{U}} v_{u}^{0} \in \mathbb{R}^{+} .
$$

\subsection{Variables}

Variables taken from Section 2 include $f_{c, u_{1}, u_{2}}, p_{c, u}, x_{u}, K$, and $V$. 
The status of the three characteristics in every unit after the re-balancing is stored in $s_{c, u} \in\left[0, S_{\max }\right]$. To model the continuous piecewise linear functions necessary to determine a status, the incremental method [22] is used with auxiliary variables $w_{c, u, i}$ and $\delta_{c, u, i}$, $i \in\left\{1, \ldots, S_{\max }\right\}$. As the maximum status $s_{u}^{\max }$ and minimum status $s_{u}^{\min }$ of each unit have to be determined, the maximum and minimum functions have to be linearized to fit into the MILP model. Therefore, the following auxiliary variables are added: $z_{u, 1}^{\max }, z_{u, 2}^{\max }$, $z_{u, 1}^{\min }, z_{u, 2}^{\min }, \lambda_{u}^{\max }, \lambda_{u}^{\min }$. The cost $k_{u}$ and efficiency $v_{u}$ of each unit is computed similarly to $s_{c, u}$ with auxiliary variables $w_{u, i}^{k}, \delta_{u, i}^{k}, w_{u, i}^{v}, \delta_{u, i}^{v}, i \in\left\{1, \ldots, S_{\max }\right\}$. Finally, $\theta_{u, b} \in\{0,1\}$ tells us if the minimum status of a unit $u$ is higher than $b$. All new variables are listed in Table 2.

Table 2. Additional variables in the continuous model.

\begin{tabular}{|c|c|c|}
\hline Name & Range & Description \\
\hline$s_{c, u}$ & {$\left[0, S_{\max }\right]$} & status of characteristic $c$ in unit $u$ after re-balancing \\
\hline$w_{c, u, i}$ & $\{0,1\}$ & Is $s_{c, u}$ at least $i-1$ (yes $/$ no)? \\
\hline$\delta_{c, u, i}$ & {$[0,1]$} & position of $s_{c, u}$ on the interval $[i-1, i]$ \\
\hline$s_{u}^{\max }$ & {$\left[0, S_{\max }\right]$} & maximum status of unit $u$ "after" \\
\hline$\lambda_{u}^{\max }$ & {$\left[0, S_{\max }\right]$} & $\max \left(s_{\mathrm{ar}, u}, s_{\mathrm{pe}, u}\right)$ \\
\hline$z_{u, 1}^{\max }$ & $\{0,1\}$ & Is $s_{\mathrm{pe}, u} \geq s_{\mathrm{ar}, u}(\mathrm{yes} / \mathrm{no}) ?$ \\
\hline$z_{u, 2}^{\max }$ & $\{0,1\}$ & Is $s_{\mathrm{eq}, u} \geq \lambda_{u}^{\max }(\mathrm{yes} / \mathrm{no}) ?$ \\
\hline$s_{u}^{\min }$ & {$\left[0, S_{\max }\right]$} & minimum status of unit $u$ "after" \\
\hline$\lambda_{u}^{\min }$ & {$\left[0, S_{\max }\right]$} & $\min \left(s_{\mathrm{ar}, u}, s_{\mathrm{pe}, u}\right)$ \\
\hline$z_{u, 1}^{\min }$ & $\{0,1\}$ & Is $s_{\mathrm{pe}, u} \leq s_{\mathrm{ar}, u}($ yes $/ \mathrm{no}) ?$ \\
\hline$z_{u, 2}^{u, 1}$ & $\{0,1\}$ & Is $s_{\mathrm{eq}, u} \leq \lambda_{u}^{\min }(\mathrm{yes} / \mathrm{no}) ?$ \\
\hline$k_{u}^{u, 2}$ & $\mathbb{R}^{+}$ & cost of unit $u$ "after" \\
\hline$w_{u, i}^{k}$ & $\{0,1\}$ & Is $k_{u}$ at least $g_{i-1}^{k}($ yes $/$ no $) ?$ \\
\hline$\delta_{u, i}^{k, i}$ & {$[0,1]$} & position of $k_{u}$ on the interval $\left[g_{i-1}^{k}, g_{i}^{k}\right]$ \\
\hline$v_{u}$ & $\mathbb{R}^{+}$ & efficiency of unit $u$ "after" \\
\hline$w_{u, i}^{v}$ & $\{0,1\}$ & Is $v_{u}$ at least $g_{i-1}^{v}($ yes $/$ no $) ?$ \\
\hline$\delta_{u, i}^{v, i}$ & {$[0,1]$} & position of $v_{u}$ on the interval $\left[g_{i-1}^{v}, g_{i}^{v}\right]$ \\
\hline$\theta_{u, b}$ & $\{0,1\}$ & Is $s_{u}^{\min }>b($ yes $/ \mathrm{no}) ?$ \\
\hline
\end{tabular}

\subsection{Objective and Constraints}

The primary goal is to maximize the total efficiency after re-balancing the units. As a secondary objective, the required shipping costs are to be minimized:

$$
\max V-\varepsilon \sigma
$$

with

$$
\sigma=\sum_{\substack{c \in C^{\prime} \\ u, u_{1} \in U}} \kappa_{c} \cdot \gamma_{r_{u}, r_{u_{1}}} \cdot d\left(u, u_{1}\right) \cdot f_{c, u, u 1},
$$

and $\varepsilon$ being a sufficiently small value (we use $\varepsilon:=10^{-6}$ ).

After the re-balancing, the following conditions must hold. The total efficiency is computed by adding up the efficiency of the individual units:

$$
V=\sum_{u \in \mathcal{U}} v_{u}
$$

The total costs are computed similarly:

$$
K=\sum_{u \in \mathcal{U}} k_{u}
$$


The budget is limited. The re-balanced units must not be more expensive than "before":

$$
K \leq K^{0} .
$$

A closed unit has to be emptied completely:

$$
p_{c, u} \leq x_{u}, \quad \forall u \in \mathcal{U}, c \in \mathcal{C} .
$$

An open unit must have a minimum amount of any characteristic:

$$
\epsilon_{\text {open }} \cdot x_{u} \leq p_{c, u}, \quad \forall u \in \mathcal{U}, c \in \mathcal{C} .
$$

The existing units can be distributed (cannibalized) to other units:

$$
p_{c, u}^{0}=\sum_{u_{1} \in U} f_{c, u, u_{1}}, \quad p_{c, u}=\sum_{u_{0} \in U} f_{c, u_{0}, u}, \quad \forall u \in \mathcal{U}, c \in \mathcal{C} .
$$

The status "after" is modeled as a continuous piecewise linear function of $p_{c, u}$ :

$$
\begin{gathered}
w_{c, u, i} \geq \delta_{c, u, i}, \quad \forall u \in \mathcal{U}, c \in \mathcal{C}, i \in\left\{1, \ldots, S_{\max }\right\}, \\
\delta_{c, u, i} \geq w_{c, u, i+1}, \quad \forall u \in \mathcal{U}, c \in \mathcal{C}, i \in\left\{1, \ldots, S_{\max }-1\right\}, \\
p_{c, u}=g_{c, 1} \delta_{c, u, 1}+\sum_{i=2}^{S_{\max }}\left(g_{c, i}-g_{c, i-1}\right) \delta_{c, u, i}, \quad \forall u \in \mathcal{U}, c \in \mathcal{C}, \\
s_{c, u}=\sum_{i=1}^{S_{\max }} \delta_{c, u, i}, \quad \forall u \in \mathcal{U}, c \in \mathcal{C} .
\end{gathered}
$$

To find the maximum status of a unit among its three characteristics "after", the maximum of two arbitrary statuses (w.l.o.g. we choose armor and personnel) is identified first and saved as $\lambda_{u}^{\max }$ :

$$
\begin{gathered}
\lambda_{u}^{\max } \geq s_{\mathrm{ar}, u}, \quad \forall u \in \mathcal{U}, \\
\lambda_{u}^{\max } \geq s_{\mathrm{pe}, u}, \quad \forall u \in \mathcal{U}, \\
\lambda_{u}^{\max } \leq s_{\mathrm{ar}, u}+S_{\max } z_{u, 1}^{\max }, \quad \forall u \in \mathcal{U}, \\
\lambda_{u}^{\max } \leq s_{\mathrm{pe}, u}+S_{\max }\left(1-z_{u, 1}^{\max }\right), \quad \forall u \in \mathcal{U} .
\end{gathered}
$$

Thereupon, the maximum of $\lambda_{u}^{\max }$ and the third status (in our case: equipment) is determined to find the "true" maximum $s_{u}^{\max }$ :

$$
\begin{gathered}
s_{u}^{\max } \geq \lambda_{u}^{\max }, \quad \forall u \in \mathcal{U}, \\
s_{u}^{\max } \geq s_{\mathrm{eq}, u}, \quad \forall u \in \mathcal{U}, \\
s_{u}^{\max } \leq \lambda_{u}^{\max }+S_{\max } z_{u, 2}^{\max }, \quad \forall u \in \mathcal{U}, \\
s_{u}^{\max } \leq s_{\text {eq }, u}+S_{\max }\left(1-z_{u, 2}^{\max }\right), \quad \forall u \in \mathcal{U} .
\end{gathered}
$$

The minimum status $s_{u}^{\min }$ of a unit is determined similarly:

$$
\begin{gathered}
\lambda_{u}^{\min } \leq s_{\mathrm{ar}, u}, \quad \forall u \in \mathcal{U}, \\
\lambda_{u}^{\min } \leq s_{\mathrm{pe}, u}, \quad \forall u \in \mathcal{U}, \\
\lambda_{u}^{\min } \geq s_{\mathrm{ar}, u}-S_{\max } z_{u, 1}^{\min }, \quad \forall u \in \mathcal{U}, \\
\lambda_{u}^{\min } \geq s_{\mathrm{pe}, u}-S_{\max }\left(1-z_{u, 1}^{\min }\right), \quad \forall u \in \mathcal{U}, \\
s_{u}^{\min } \leq \lambda_{u}^{\min }, \quad \forall u \in \mathcal{U},
\end{gathered}
$$




$$
\begin{gathered}
s_{u}^{\min } \leq s_{\mathrm{eq}, u}, \quad \forall u \in \mathcal{U}, \\
s_{u}^{\min } \geq \lambda_{u}^{\min }-S_{\max } z_{u, 2}^{\min }, \quad \forall u \in \mathcal{U}, \\
s_{u}^{\min } \geq s_{\text {eq }, u}-S_{\max }\left(1-z_{u, 2}^{\min }\right), \quad \forall u \in \mathcal{U} .
\end{gathered}
$$

The cost and efficiency of a unit are continuous piecewise linear functions of $s_{u}^{\max }$ and $s_{u}^{\min }$, respectively:

$$
\begin{gathered}
w_{u, i}^{k} \geq \delta_{u, i}^{k}, \quad \forall u \in \mathcal{U}, i \in\left\{1, \ldots, S_{\max }\right\}, \\
\delta_{u, i}^{k} \geq w_{u, i+1}^{k}, \quad \forall u \in \mathcal{U}, i \in\left\{1, \ldots, S_{\max }-1\right\}, \\
s_{u}^{\max }=\sum_{i=1}^{S_{\max }} \delta_{u, i^{\prime}}^{k} \quad \forall u \in \mathcal{U}, c \in \mathcal{C}, \\
k_{u}=g_{1}^{k} \delta_{u, 1}^{k}+\sum_{i=2}^{S_{\max }}\left(g_{i}^{k}-g_{i-1}^{k}\right) \delta_{u, i}^{k} \quad \forall u \in \mathcal{U}, c \in \mathcal{C}, \\
w_{u, i}^{v} \geq \delta_{u, i^{\prime}}^{v}, \quad \forall u \in \mathcal{U}, i \in\left\{1, \ldots, S_{\max }\right\}, \\
\delta_{u, i}^{v} \geq w_{u, i+1}^{v}, \quad \forall u \in \mathcal{U}, i \in\left\{1, \ldots, S_{\max }-1\right\}, \\
s_{u}^{\min }=\sum_{i=1}^{S_{\max }} \delta_{u, i}^{v}, \quad \forall u \in \mathcal{U}, c \in \mathcal{C}, \\
v_{u}=g_{1}^{v} \delta_{u, 1}^{v}+\sum_{i=2}^{S_{\max }}\left(g_{i}^{v}-g_{i-1}^{v}\right) \delta_{u, i}^{v}, \quad \forall u \in \mathcal{U}, c \in \mathcal{C} .
\end{gathered}
$$

The minimum status of a unit may or may not exceed a given level:

$$
\begin{gathered}
S_{\max } \theta_{u, b} \leq S_{\max }+s_{u}^{\min }-b, \forall u \in \mathcal{U}, b \in \mathcal{B}, \\
s_{u}^{\min }-b \leq S_{\max } \theta_{u, b}-\varepsilon, \quad \forall u \in \mathcal{U}, b \in \mathcal{B} .
\end{gathered}
$$

Every region must contain a certain number of units with their lowest status between given levels:

$$
\underline{n}_{r}(b) \leq \sum_{\substack{u \in U: \\ r_{u}=r}} \theta_{u, b} \leq \bar{n}_{r}(b), \quad \forall r \in \mathcal{R}, b \in \mathcal{B} .
$$

Shipping costs are not allowed to exceed $\sigma_{\max }$ :

$$
\sum_{\substack{c \in C_{,} \\ u, u_{1} \in U}} \kappa_{c} \cdot \gamma_{r_{u}, r_{u_{1}}} \cdot d\left(u, u_{1}\right) \cdot f_{c, u, u 1} \leq \sigma_{\max } .
$$

The model (C) consists of (31)-(70).

\section{Computational Results}

\subsection{A Demonstrator Example}

We consider an instance with $U=5$ units, $C=3$ characteristics (armor, personnel, and equipment), and $S=8$ statuses. The ratings before the re-balancing can be found in the following matrix:

$$
\left(p_{c, u}^{0}\right)_{c, u}:=\underset{\text { eq }}{\text { pe }}\left(\begin{array}{ccccc}
1 & 2 & 3 & 4 & 5 \\
0.2 & 0.7 & 0.5 & 0.9 & 0.1 \\
0.5 & 0.3 & 0.7 & 0.8 & 0.4 \\
0.8 & 0.5 & 0.4 & 0.9 & 0.6
\end{array}\right) .
$$

The coordinates and region of each unit can be found in Table 3. 
Table 3. Coordinates and region of units.

\begin{tabular}{cccc}
\hline $\boldsymbol{u}$ & $\boldsymbol{X}_{\boldsymbol{u}}$ & $\boldsymbol{Y}_{\boldsymbol{u}}$ & $\boldsymbol{r}_{\boldsymbol{u}}$ \\
\hline 1 & 1 & 1 & 1 \\
2 & 2 & 3 & 1 \\
3 & 5 & 5 & 2 \\
4 & 6 & 5 & 2 \\
5 & 1 & 9 & 3 \\
\hline
\end{tabular}

Shipping costs are defined as follows:

$$
\left(\gamma_{r_{1}, r_{2}}\right)_{r_{1}, r_{2}}:=\begin{array}{ccc}
1 & 2 & 3 \\
2 & 3
\end{array}\left(\begin{array}{ccc}
1 & 1.5 & 2.5 \\
1.5 & 1 & 2 \\
2.5 & 2 & 1
\end{array}\right), \kappa_{\mathrm{ar}}=2, \kappa_{\mathrm{pe}}=0.3, \kappa_{\mathrm{eq}}=1.2 .
$$

Total shipping costs may not exceed $\sigma_{\max }=5$. In the following, this instance is modeled and solved in both the basic and the continuous model.

\subsubsection{Basic Model}

The interval range for the statuses of each characteristic are as follows:

$$
\begin{aligned}
& \begin{array}{llllllll}
1 & 2 & 3 & 4 & 5 & 6 & 7 & 8
\end{array}
\end{aligned}
$$

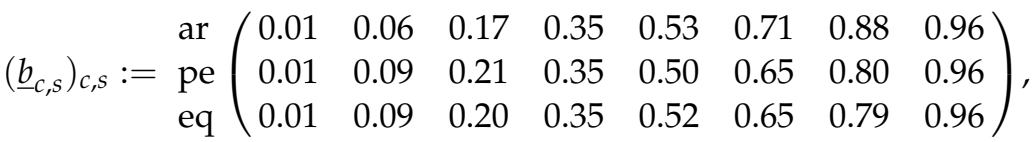

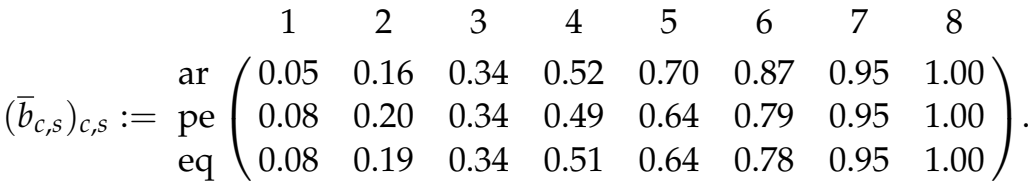

As an example, Figure 2 shows how the personnel ratings of the five units get converted into statuses.

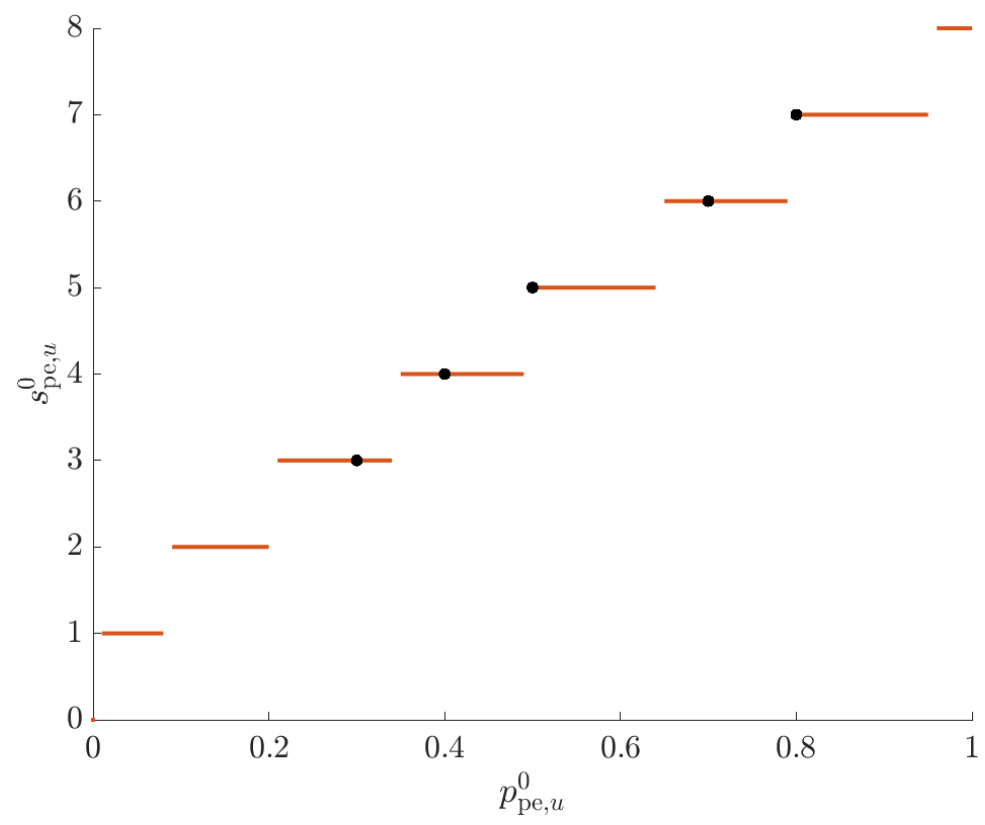

Figure 2. Conversion of personnel rating into personnel status in the basic model. 
Furthermore, cost coefficients

$$
\left(k_{s}\right)_{s}=(0.20,0.32,0.44,0.53,0.69,0.79,0.87,0.98)
$$

and efficiency coefficients

$$
\left(v_{s}\right)_{s}=(0.02,0.14,0.28,0.41,0.59,0.71,0.83,0.92)
$$

are given. Additionally, the following restrictions are enforced:

$$
\begin{gathered}
\underline{n}_{1,3}=2, \underline{n}_{1,5}=1, \underline{n}_{2,7}=1 ; \\
\bar{n}_{1,7}=1, \bar{n}_{3,5}=0 .
\end{gathered}
$$

By inspection of matrix $\left(p_{c, u}^{0}\right)_{c, u}$ one can see that the statuses of the units are as follows:

$$
\begin{array}{lllll}
1 & 2 & 3 & 4 & 5 \\
\text { lowest } \\
\text { highest }
\end{array}\left(\begin{array}{lllll}
3 & 3 & 4 & 7 & 2 \\
7 & 5 & 6 & 7 & 5
\end{array}\right) .
$$

The total costs are $K^{0}=3.91$, and the efficiency is $V^{0}=1.94$.

Running the above model with these input data leads to the following solution:

$$
\left(p_{c, u}\right)_{c, u}:=\underset{\text { eq }}{\text { ar }}\left(\begin{array}{ccccc}
1 & 2 & 3 & 4 & 5 \\
0.35 & 0.55 & 0.37 & 0.96 & 0.17 \\
0.35 & 0.50 & 0.49 & 0.96 & 0.40 \\
0.77 & 0.52 & 0.35 & 0.96 & 0.60
\end{array}\right) .
$$

By inspection of matrix $\left(p_{c, u}\right)_{c, u}$ one can see that the statuses of units are as follows:

$$
\begin{array}{lllll}
1 & 2 & 3 & 4 & 5 \\
\text { highest } & \left(\begin{array}{lllll}
4 & 5 & 4 & 8 & 3 \\
6 & 5 & 4 & 8 & 5
\end{array}\right) .
\end{array}
$$

The efficiency has increased to $V=2.61$ while the total costs have sunk to $K=3.68$. The shipping costs for the re-balancing operation add up to 2.822 .

\subsubsection{Continuous Model}

Defining $\mathcal{B}:=\{3,5,7\}$, the regional restrictions from Section 4.1.1 can be copied. The breakpoints of the piecewise linear functions are derived from the upper bounds $\bar{b}_{c, s}$ of the status intervals in the basic model:

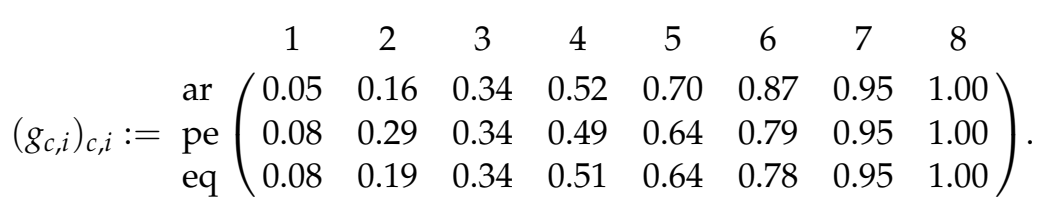

To determine the cost and efficiency of a unit, we use

$$
\begin{aligned}
& g^{k}=(0.20,0.32,0.44,0.53,0.69,0.79,0.87,0.98), \\
& g^{v}=(0.02,0.14,0.28,0.41,0.59,0.71,0.83,0.92) .
\end{aligned}
$$


Converting $\left(p_{c, u}^{0}\right)_{c, u}$ into status values yields

$$
\left(s_{c, u}^{0}\right)_{c, u}:=\underset{\text { pe }}{\text { ar }}\left(\begin{array}{ccccc}
1 & 2 & 3 & 4 & 5 \\
2.222 & 5 & 3.889 & 6.375 & 1.455 \\
4.067 & 2.2 & 5.4 & 6.706 & 3.4 \\
6.118 & 3.941 & 3.353 & 6.063 & 4.692
\end{array}\right) .
$$

Before the optimization, total costs add up to $K^{0}=3.707$. The total efficiency is $V^{0}=1.457$.

Re-balancing offers the following solution:

$$
\left(p_{c, u}\right)_{c, u}:=\underset{\text { eq }}{\text { ar }}\left(\begin{array}{ccccc}
1 & 2 & 3 & 4 & 5 \\
0.34 & 0.70 & 0.20 & 1 & 0.16 \\
0.34 & 0.64 & 0.34 & 1 & 0.38 \\
0.66 & 0.64 & 0.30 & 1 & 0.60
\end{array}\right),
$$

corresponding to the following statuses:

$$
\left(s_{c, u}\right)_{c, u}:=\underset{\text { pe }}{\operatorname{ar}}\left(\begin{array}{ccccc}
1 & 2 & 3 & 4 & 5 \\
3 & 5 & 2.222 & 8 & 2 \\
3 & 5 & 2.733 & 8 & 3.356 \\
5.143 & 5 & 2.733 & 8 & 4.692
\end{array}\right) .
$$

Total costs have fallen to $K=3.423$ and total efficiency has been raised to $V=2.101$. Shipping costs amount to $\sigma=4.672$.

An overview of the location and initial strength of each unit together with the effect of the re-balancing is given in Figure 3.
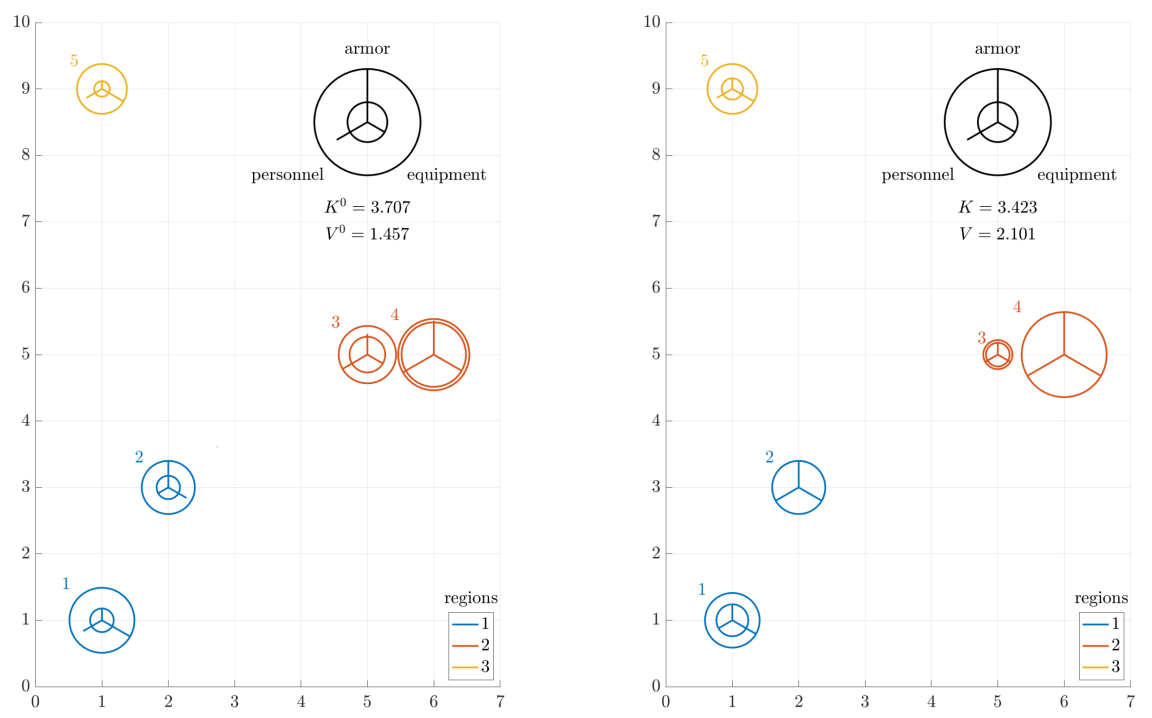

Figure 3. Location and strength of units prior to (left) and after optimization (right).

\subsection{Comparison of Solvers}

In this section, we want to focus on the continuous model. After formulating the model as a mixed-integer linear programming problem as described in Section 3, numerical solvers can be used to find feasible solutions and - in the best case-verify that the provided solution is optimal. For our computations, we used an Apple MacBook Pro laptop running an Intel Core i7 at $3.10 \mathrm{GHz}$ clock speed and 16 GB RAM. All instances were provided for the algebraic modeling language AMPL (version 20190927). The following solvers were available: CPLEX 12.9, Gurobi 8.1, Xpress 8.6. 
To identify the best among the three solvers for instances of our model, we compared their performance on a variety of instances. For a fixed maximum status $\left(S_{\max }=5\right)$ and number of regions $(R=9)$, we varied the number of units between 5 and 30, generating 20 instances each. Coordinates were randomly assigned on a $100 \times 100$ square area. Regions were determined by dividing the area into thirds horizontally and vertically. Shipping costs were generated so that $\gamma_{r_{1}, r_{2}}=\gamma_{r_{2}, r_{1}}$ for all $r_{1}, r_{2} \in \mathcal{R}$ and $\kappa_{\mathrm{ar}} \geq \kappa_{\mathrm{eq}} \geq \kappa_{\mathrm{pe}}$. Regional restrictions were not enforced.

For this sample, Gurobi returned the best results. With Gurobi, instances were solved fastest or, when reaching the maximum computation time of one hour, left the smallest relative gaps between the best feasible solution found and the best upper bound. Average solution times for each solver can be seen in Figure 4. In total, Gurobi needed 684,422 s to solve all instances with the remaining gaps summing up to $147 \%$. CPLEX needed 704,981 s and left a total sum of gaps of $207 \%$. Solving all instances with Xpress took the longest time with $809,152 \mathrm{~s}$ and summed gaps of $263 \%$. Consequently, we used Gurobi for all following computations. Note that although many instances exceeded the maximum computation time of one hour, Gurobi left gaps no bigger than $6.4 \%$. In most cases, solutions with a remaining gap of $<1 \%$ could be found in under a minute.

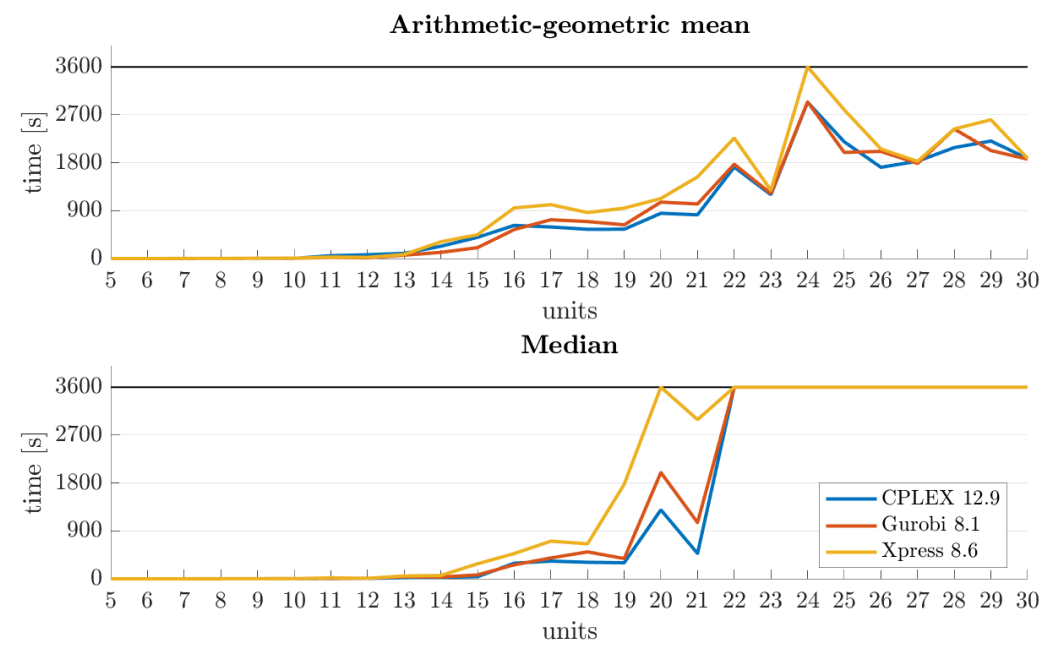

Figure 4. Average solution times and gaps of the three solvers depending of the number of units.

\subsection{Analyzing Solver Performance}

As we can see in Figure 4, increasing the number of units also increases the average computation time. In order to gain more detailed computation results, 20 instances for $U=31, \ldots, 40$ were additionally solved with Gurobi. Solving all new instances took a total of $634,922 \mathrm{~s}$. The results are displayed in Figure 5.

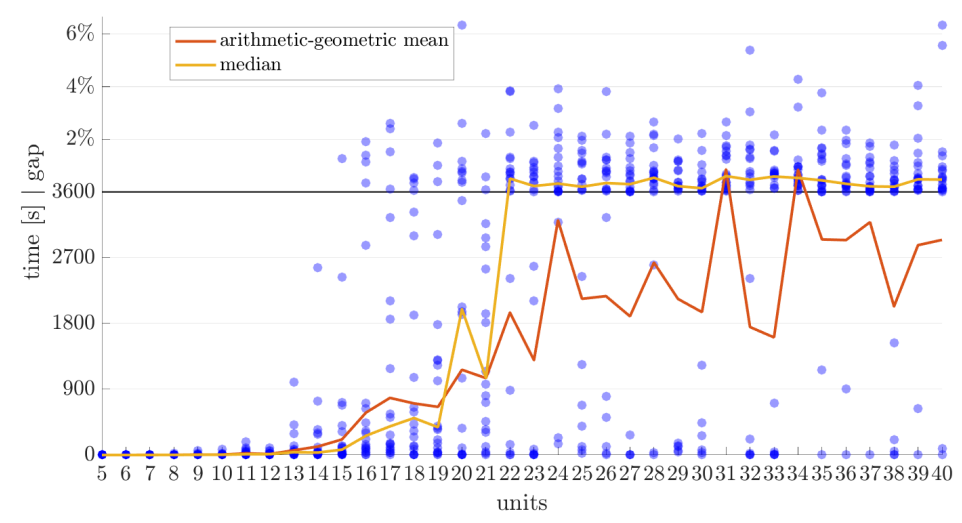

Figure 5. Solution time depending of the number of units. 
For 22 and more units, the optimal solution for the median instance could not be provided within an hour. However, outliers seem to occur independent of the amount of units: a 40-unit instance was solved in $5 \mathrm{~s}$. The average gap was highest for $U=40$ with $1.02 \%$.

\subsection{A Real-World Example}

Goal of this paper is the re-balancing of $U=75$ army units which are distributed over $R=12$ regions.

\subsubsection{Basic Model}

For a given status quo, each characteristic of every unit can be assigned to one of $S=5$ statuses with the following interval ranges:

$$
\begin{aligned}
& \left(\underline{b}_{c, s}\right)_{c, s}:=\begin{array}{ccccc}
1 & 2 & 3 & 4 & 5 \\
\text { eq } & \text { eq }
\end{array}\left(\begin{array}{ccccc}
0.01 & 0.16 & 0.65 & 0.90 & 0.98 \\
0.01 & 0.05 & 0.12 & 0.36 & 0.81 \\
0.01 & 0.10 & 0.35 & 0.63 & 0.96
\end{array}\right), \\
& \left(\bar{b}_{c, s}\right)_{c, s}:=\underset{\text { pe }}{\text { ar }}\left(\begin{array}{ccccc}
1 & 2 & 3 & 4 & 5 \\
0.15 & 0.64 & 0.89 & 0.97 & 1.00 \\
0.04 & 0.11 & 0.35 & 0.80 & 1.00 \\
0.09 & 0.34 & 0.62 & 0.95 & 1.00
\end{array}\right) .
\end{aligned}
$$

Cost and efficiency coefficients are chosen as follows:

$$
\begin{aligned}
& \left(k_{s}\right)_{s}=(0.27,0.51,0.68,0.81,0.94), \\
& \left(v_{s}\right)_{s}=(0.16,0.38,0.49,0.76,0.89) .
\end{aligned}
$$

The regional restrictions are listed in Table 4.

Table 4. Regional restrictions for a 75-unit instance.

\begin{tabular}{cccccccccccc}
\hline $\boldsymbol{r}$ & $\boldsymbol{s}$ & $\underline{\boldsymbol{n}}_{r, \boldsymbol{s}}$ & $\overline{\boldsymbol{n}}_{\boldsymbol{r}, \boldsymbol{s}}$ & $\boldsymbol{r}$ & $\boldsymbol{s}$ & $\underline{\boldsymbol{n}}_{r, \boldsymbol{s}}$ & $\overline{\boldsymbol{n}}_{\boldsymbol{r}, \boldsymbol{s}}$ & $\boldsymbol{r}$ & $\boldsymbol{s}$ & $\underline{\boldsymbol{n}}_{r, s}$ & $\overline{\boldsymbol{n}}_{\boldsymbol{r}, \boldsymbol{s}}$ \\
\hline 3 & 3 & 4 & 4 & 9 & 3 & 8 & 8 & 11 & 3 & 4 & 4 \\
3 & 4 & 2 & 2 & 9 & 4 & 3 & 3 & 11 & 4 & 2 & 2 \\
7 & 3 & 8 & 8 & 10 & 3 & 4 & 4 & 12 & 3 & 4 & 4 \\
7 & 4 & 3 & 3 & 10 & 4 & 2 & 2 & 12 & 4 & 2 & 2 \\
\hline
\end{tabular}

Furthermore, let $\kappa_{\mathrm{ar}}=0.9, \kappa_{\mathrm{pe}}=0.3, \kappa_{\mathrm{eq}}=0.8$.

The total costs prior to the optimization sum up to $K^{0}=56.37$, resulting in a total efficiency of $V^{0}=37.15$.

Re-balancing increases the total efficiency to $V=50.84$ (increase of $36.9 \%$ ) while lowering the cost to $K=56.27$.

\subsubsection{Continuous Model}

For a given status quo, each characteristic of every unit can be assigned to a status $\in[0,5]$. The breakpoints for the piecewise linear functions of armor, personnel, and equipment are defined by the test functions $x \mapsto x^{2}, x \mapsto \sqrt{x}$, and $x \mapsto x$, respectively. For example, a square function used for the pe characteristic would impose a quadratic benefit from adding more personnel to a unit, which resembles to Lanchester's square law, see in [23]. The breakpoints are given as follows: 


$$
\left(g_{c, i}\right)_{c, i}:=\begin{array}{ccccc}
1 & 2 & 3 & 4 & 5 \\
\text { eq } & \text { pe }
\end{array}\left(\begin{array}{ccccc}
0.45 & 0.63 & 0.77 & 0.89 & 1.00 \\
0.04 & 0.16 & 0.36 & 0.64 & 1.00 \\
0.20 & 0.40 & 0.60 & 0.80 & 1.00
\end{array}\right) .
$$

The corresponding piecewise linear functions are shown in Figure 6.

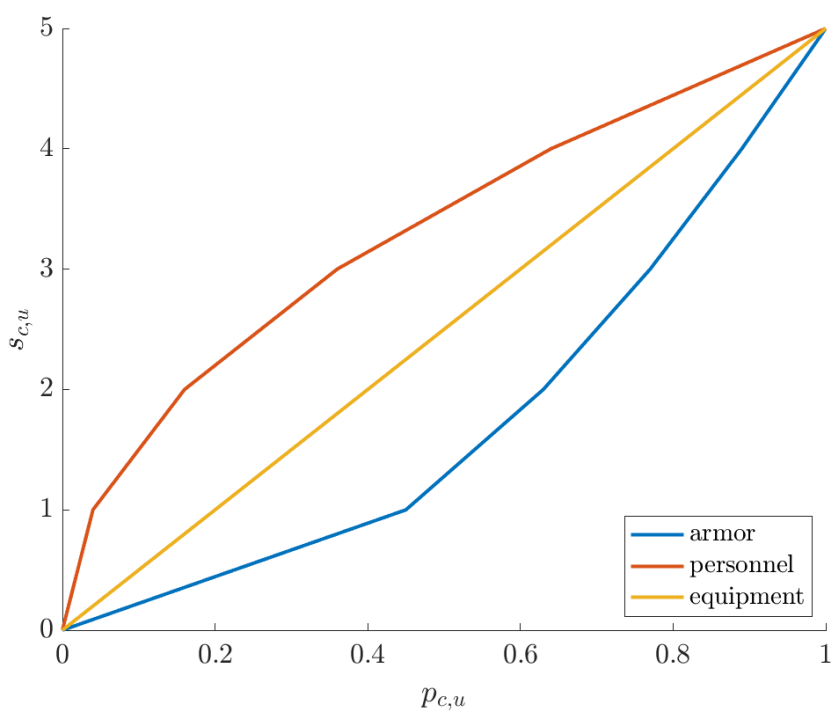

Figure 6. Piecewise linear functions to determine the status of a characteristic.

Let $g^{k}=\left(k_{s}\right)_{s}$ and $g^{v}=\left(v_{s}\right)_{s}$ from the basic model. All other parameters are taken over. An overview of the location and initial strength of each unit is given in Figure 7.

Before the re-balancing, the total efficiency equals $V^{0}=32.449$ with total costs of $K^{0}=50.971$. Afterwards, the efficiency is increased to $V=37.279$ (14.9\% increase) while total costs have fallen to $K=48.754$. Shipping costs for the re-balancing operations sum up to $\sigma=750.834$. As a result of the re-balancing process, six units were closed. The effects can be seen in Figure 8 .

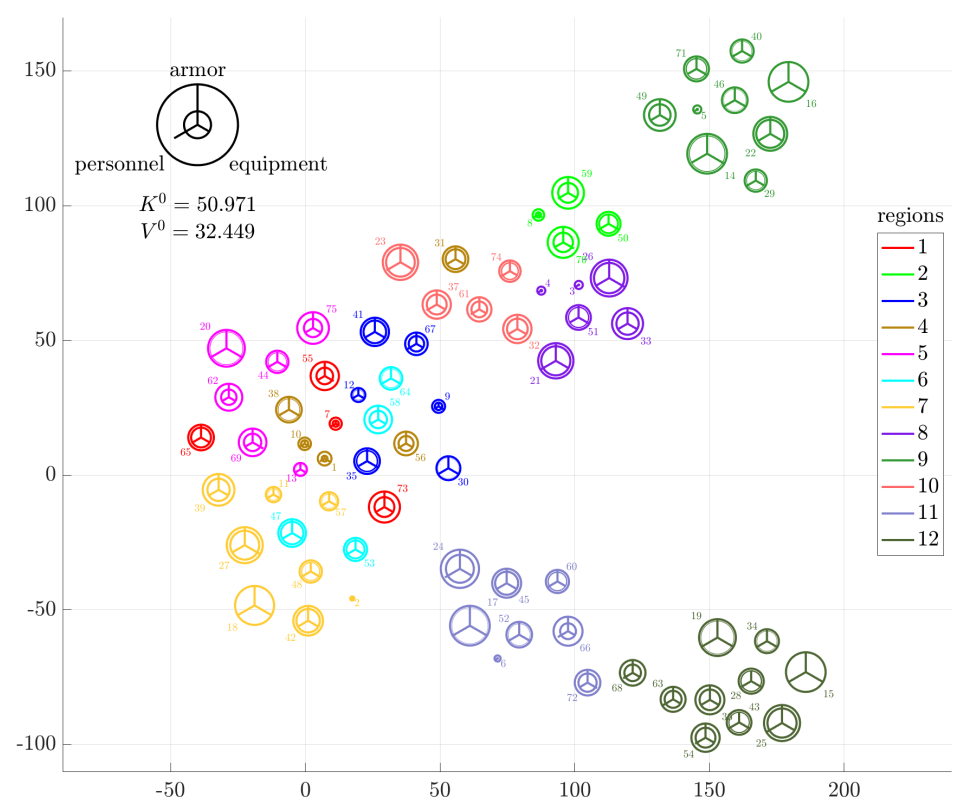

Figure 7. Location and strength of units prior to optimization. 


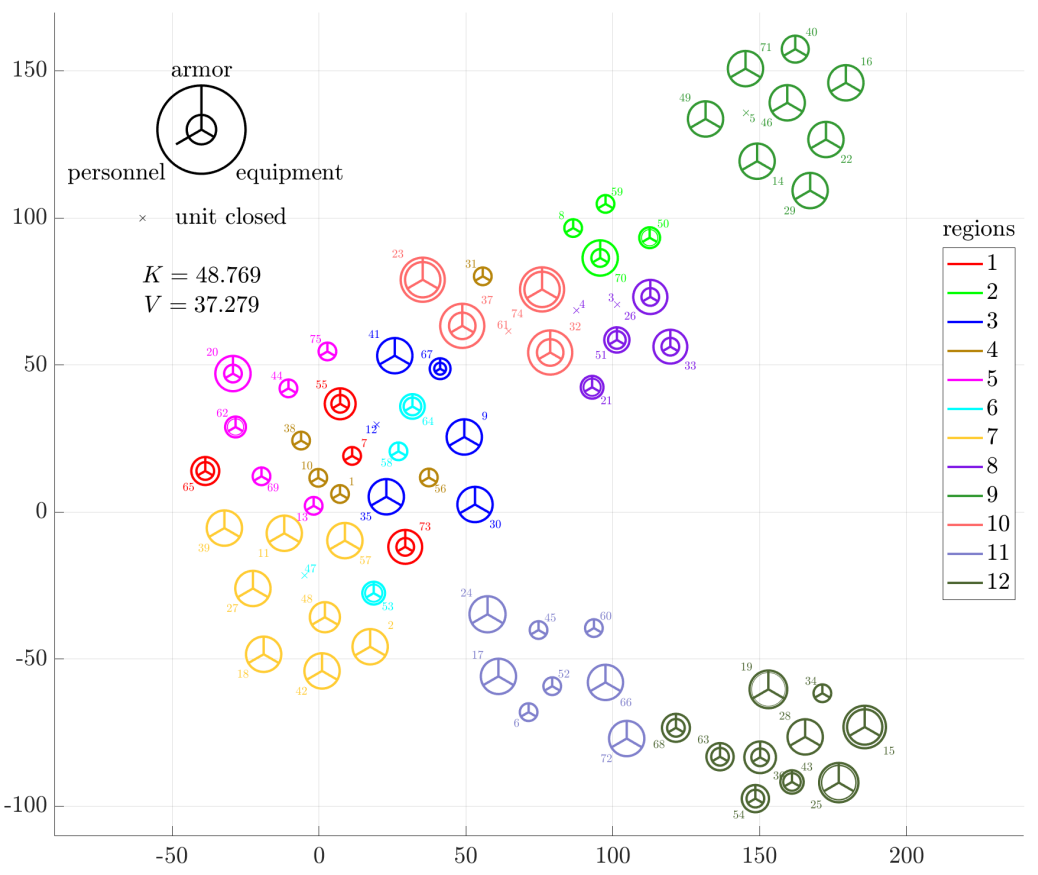

Figure 8. Location and strength of units after optimization.

The movements of each characteristic between the regions can be visualized in alluvial flow diagrams [24]. Exemplarily, the shifts of personnel are shown in Figure 9.

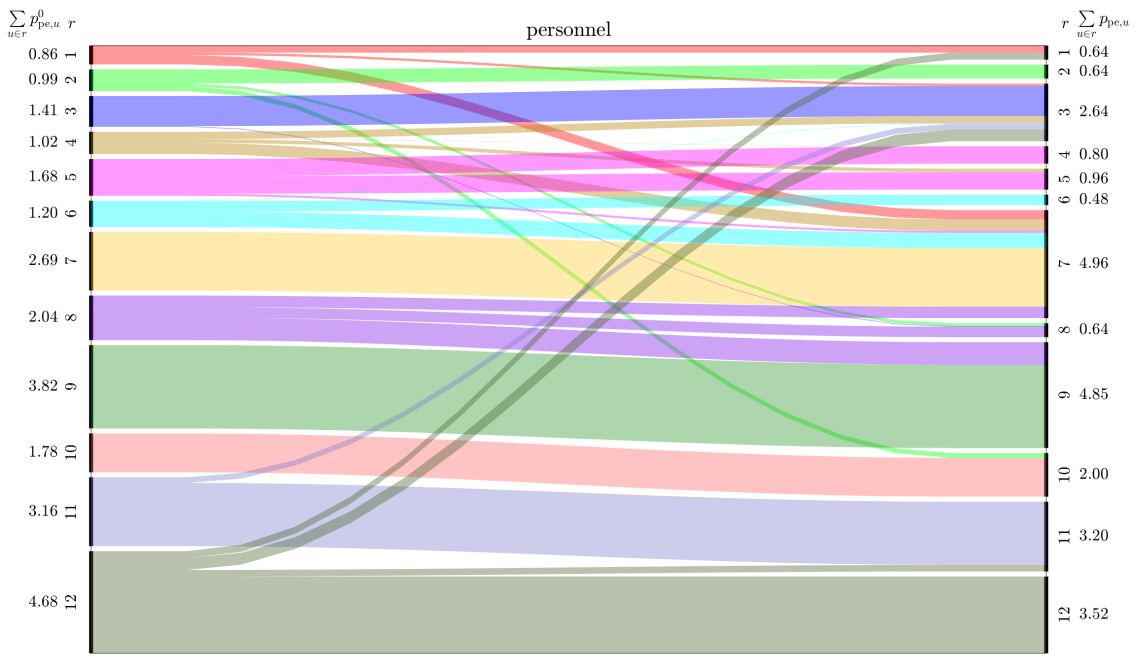

Figure 9. Alluvial flow diagram displaying shifts of personnel between regions.

\subsection{Trade-Off between Budget and Efficiency}

In our model, we demand that the total costs of all units $K$ after the re-balancing cannot exceed the total costs $K^{0}$ beforehand. This constraint could either be loosened (e.g., by allowing a maximum cost increase of 10\%) or tightened (e.g., by additionally forcing a $20 \%$ reduction of the total costs). With allowing a cost increase, one would hope for a corresponding increase in total efficiency. On the other hand, reducing the total costs will probably lessen the total efficiency. To check the effects of increasing or decreasing the maximum total costs, we reverted to the instance solved in Section 4.4.2. As we can see, the solution which maximizes efficiency includes total costs after of $K=48.754$ which are well below the total costs before $\left(K^{0}=50.971\right)$. Raising the barrier for the maximum total costs would have no effect on the optimal solution. Therefore, the remaining question is 
how far the total costs can be reduced and what impact this has on the total efficiency. For this instance, the lowest possible costs (assuming no destruction of material or dismissal of personnel) sum up to $K=46.297$ (5.0\% less compared to the "highest efficiency" solution). However, efficiency also dropped to $V=34.534$ (7.4\% decrease). To identify the behavior of costs and efficiency between these two extremes, instances were solved where the maximum costs were restricted to a value in the interval [46.30, 48.75], applying the epsilonconstraint method as described in [25]. In these computations, shipping costs were ignored to speed up the process. In total, 51 instances were solved within a time restriction of one hour each, leaving a maximum gap of $0.22 \%$. Total computation time was $109,626 \mathrm{~s}$. The results are shown in Figure 10.

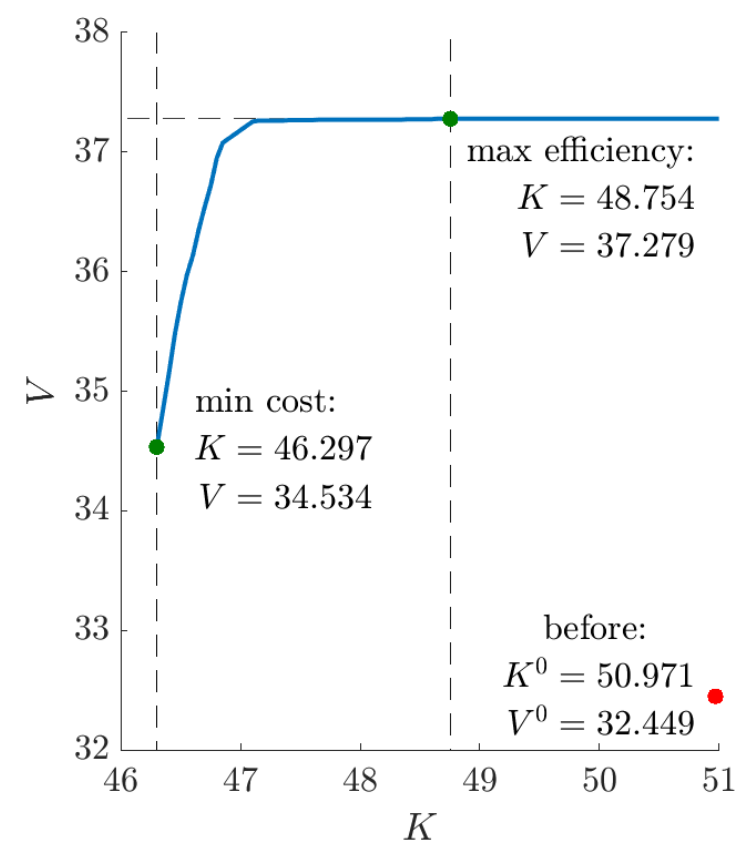

Figure 10. Trade-off between budget and efficiency for a 75-unit instance.

\section{Conclusions}

We gave a nonlinear mixed-integer model formulation for the re-balancing of resources or assets for security services. The resources or assets are moved from one unit to another. The model can be re-formulated as a mixed-integer linear program, so that it is solvable with standard numerical solvers for this problem class. It turns out that it is possible to achieve an increasing active operating strength and more efficiency with the same operational budget.

The study employed anonymized data from army units. The results indicate that re-balancing the resources has an extremely positive influence on the efficiency of the units. At the same time the total operational cost can be reduced. The way of organizing the units after the re-balancing procedure creates a force that is more effective for today's tasks.

Future research in this topic could focus on the trade-off between budget and efficiency. As the example in Figure 10 showed, total costs can be reduced significantly if one allows a minor reduction of efficiency. Consequently, one could consider a bi-objective optimization maximizing $V$ and minimizing $K$ to compute the Pareto front and to trade off between the two conflicting objectives. Shipping costs would be disregarded in this formulation as a reduction of long-term costs could be considered more important than the one-time shipping expenses. Epsilon-constraint and two-phase methods may efficiently solve this formulation. Other future projects could consider the case where certain parameters (e.g., ratings and breakpoints of the piecewise linear functions) are uncertain. Open-source solvers like $\mathrm{CBC}$ could be compared to the commercial solvers used in this paper. 
Author Contributions: Conceptualization, G.K.; methodology, R.D., A.F. and G.K.; software, R.D. and A.F.; validation, A.F. and G.K.; formal analysis, R.D.; investigation, R.D.; resources, G.K.; data curation, G.K.; writing — original draft preparation, R.D. and A.F.; writing—review and editing, G.K.; visualization, R.D.; supervision, A.F. All authors have read and agreed to the published version of the manuscript.

Funding: This research received no external funding.

Institutional Review Board Statement: Not applicable.

Informed Consent Statement: Not applicable.

Data Availability Statement: The data presented in this study are openly available in Zenodo at https:/ / doi.org/10.5281/zenodo.5579319.

Conflicts of Interest: The authors declare no conflicts of interest.

\section{References}

1. Johnson, S.E.; Peters, J.E.; Kitchens, K.E.; Martin, A.; Jordan, R.; Fischbach, J.R. A Review of the Army's Modular Force Structure; Technical Report, TR-927-2-OSD; RAND Corporation: Santa Monica, CA, USA, 2012. Available online: https://www.rand.org/ pubs/technical_reports/TR927-2.html (accessed on 7 December 2021).

2. Miller, J.G. Living Systems; McGraw Hill: New York, NY, USA, 1978.

3. Bailey, K.D. Living Systems Theory and Social Entropy Theory. Syst. Res. Behav. Sci. 2006, 23, 291-300. [CrossRef]

4. Takahashi, A.; Tsuzuki, Y. Rebalancing static super-replications. Int. J. Financ. Eng. 2017, 4, 1750003. [CrossRef]

5. Wagalath, L. Modelling the rebalancing slippage of leveraged exchange-traded funds. Quant. Financ. 2014, 14, 1503-1511. [CrossRef]

6. Wang, M.; Fengmin, X.; Guan, W. Sparse portfolio rebalancing model based on inverse optimization. Optim. Methods Softw. 2014, 29, 207-309. [CrossRef]

7. Cruz, F.; Subramanian, A.; Bruck, B.P.; Iori, M. A heuristic algorithm for a single vehicle static bike sharing rebalancing problem. Comput. Oper. Res. 2017, 79, 19-33. [CrossRef]

8. Schuijbroek, J.; Hampshire, R.C.; van Hoeve, W.J. Inventory rebalancing and vehicle routing in bike sharing systems. Eur. J. Oper. Res. 2017, 257, 992-1004. [CrossRef]

9. Boyaci, B.; Zografos, K.G.; Geroliminis, N. An optimization framework for the development of efficient one-way car-sharing systems. Eur. J. Oper. Res. 2015, 240, 718-733. [CrossRef]

10. Bruglieria, M.; Colorni, A.; Lue, A. The vehicle relocation problem for the one-way electric vehicle sharing: An application to the Milan case. Procedia Soc. Behav. Sci. 2014, 111, 18-27. [CrossRef]

11. Maynard, A.; Smallwood, A.; Wohar, M.E. Long Memory Regressors and Predictive Testing: A Two-stage Rebalancing Approach. Econom. Rev. 2013, 32, 318-360. [CrossRef]

12. Aggarwal, G.; Motwani, R.; Zhu, A. The load rebalancing problem. J. Algorithms 2006, 60, 42-59. [CrossRef]

13. Sen, S.; Tarjan, R.E.; Kim, D.H.K. Deletion without rebalancing in binary search trees. ACM Trans. Algorithms 2016, $12,1-31$. [CrossRef]

14. Belikovetsky, S.; Tamir, T. Load rebalancing games in dynamic systems with migration costs. Theor. Comput. Sci. 2016, 622, 16-33. [CrossRef]

15. Sathe, A.; Miller-Hooks, E. Optimizing Location and Relocation of Response Units in Guarding Critical Facilities. Transp. Res. Rec. J. Transp. Res. Board 2005, 1923, 127-136. [CrossRef]

16. Gao, X.; Cao, C. Multi-commodity rebalancing and transportation planning considering traffic congestion and uncertainties in disaster response. Comput. Ind. Eng. 2020, 149, 106782. [CrossRef]

17. Liu, Y.; Yuan, Y.; Li, Y.; Pang, H. A Chance Constrained Programming Model for Reliable Emergency Vehicles Relocation Problem. Procedia Soc. Behav. Sci. 2013, 96, 671-682. [CrossRef]

18. Paul, N.R.; Lunday, B.J.; Nurre, S.G. A multiobjective, maximal conditional covering location problem applied to the relocation of hierarchical emergency response facilities. Omega 2017, 66, 147-158. [CrossRef]

19. Schwarze, S.; Voß, S. Improved load balancing and resource utilization for the skill vehicle routing problem. Optim. Lett. 2013, 7, 1805-1823. [CrossRef]

20. Schwarze, S.; Voß, S. A bicriteria skill vehicle routing problem with time windows and an application to pushback operations at airports. In Logistics Management; Springer: Cham, Switzerland, 2015; pp. 289-300.

21. Peschiera, F.; Dell, R.; Royset, J.; Haï, A.; Dupin, N.; Battaïa, O. A novel solution approach with ML-based pseudo-cuts for the Flight and Maintenance Planning problem. OR Spectr. 2021, 43, 635-664. [CrossRef]

22. Markowitz, H.M.; Manne, A.S. On the Solution of Discrete Programming Problems. Econometrica 1957, 25, 84-110. [CrossRef]

23. Lanchester, F.W. Mathematics in Warfare. World Math. 1956, 4, 2138-2157.

24. Carmeli, A. Alluvial Flow Diagram. Version 1.0.0.0. Available online: https://www.mathworks.com/matlabcentral/ fileexchange/66746-alluvial-flow-diagram (accessed on 7 December 2021).

25. Ehrgott, M. Multicriteria Optimization; Springer: Berlin/Heidelberg, Germany, 2005. 